\title{
FLOW INVARIANCE FOR DIFFERENTIAL DELAY EQUATIONS
}

\author{
NAOKI TANAKA
}

(Communicated by Pamela B. Gorkin)

\begin{abstract}
The flow invariance problem for the functional differential equation $u^{\prime}(t) \in A u(t)+F\left(u_{t}\right)$ for $t \geq 0$ with initial condition $u_{0}=\phi \in \mathfrak{D}$ is solved in a Banach space $X$, where $A$ is a quasi-dissipative operator in $X$ and $F$ is a continuous operator from a closed set $\mathfrak{D}$ in the so-called initial-history space $\mathfrak{X}$ into $X$ satisfying a dissipativity condition in the following sense: There exists $\omega_{F} \geq 0$ such that $[\phi(0)-\hat{\phi}(0), F(\phi)-F(\hat{\phi})]_{+} \leq \omega_{F}\|\phi-\hat{\phi}\|_{\mathfrak{X}}$ for $\phi, \hat{\phi} \in \mathfrak{D}$ satisfying that $\|\phi-\hat{\phi}\|_{\mathfrak{X}} \leq\|\phi(0)-\hat{\phi}(0)\|_{X}$, where $[x, \xi]_{+}=$ $\lim _{h \rightarrow 0+}\left(\|x+h \xi\|_{X}-\|x\|_{X}\right) / h$ for $x, \xi \in X$.
\end{abstract}

\section{INTRODUCTION}

This paper is devoted to flow invariance for the functional differential equation $(\mathrm{FDE} ; \phi)$

$$
u^{\prime}(t) \in A u(t)+F\left(u_{t}\right) \quad \text { for } t \geq 0, \quad \text { and } \quad u_{0}=\phi \in \mathfrak{D}
$$

in a Banach space $X$, where $A$ is a quasi-dissipative operator in $X$ and $F$ is a continuous operator from a closed set $\mathfrak{D}$ in the so-called initial-history space $\mathfrak{X}$ into $X$. The initial-history space $\mathfrak{X}$ is defined as a Banach space consisting of continuous functions from $J$ into $X$ satisfying appropriate conditions, where $J$ is an interval of the form $[-R, 0]$ or $(-\infty, 0]$, and the symbol $u_{t}$ stands for the history of $u$ up to $t$, namely, $u_{t}(\theta)=u(t+\theta)$ for $\theta \in J$. The history of the problem (FDE) is found in the paper [22], including many references.

Subtangential conditions for flow invariance are well known in the case where $A=0([13,15,26])$ and in the case where $A$ is linear ([18]).

There are at least two approaches to the solvability of the equation (FDE) under the assumption that $A$ is an $m$-quasi-dissipative operator in $X$ and $F$ is globally Lipschitz continuous from $\mathfrak{X}$ into $X$. One is the way to find a weak solution as the limit function of a family of solutions to the equation governed by Yosida approximation $(9,10])$. The other is the way to obtain a representation theorem for solutions of the equation (FDE) as a quasi-contractive semigroup on a closed set $\mathfrak{X}_{0}$ of initial values in $\mathfrak{X}$, and this method is in common use for the solvability of the equation (FDE) $([2,4,8,20,21,23,25,27,28])$.

By modifying the latter idea, Ruess 24] recently gave a solution to the longstanding open problem of finding a sufficient condition for flow invariance of solutions to (FDE). One of the significant features of his result is to weaken the

Received by the editors July 28, 2013 and, in revised form, December 6, 2013, December 25, 2013 and December 27, 2013.

2010 Mathematics Subject Classification. Primary 47J35; Secondary 47H06, 47H20.

The author was partially supported by JSPS Grant-in-Aid for Scientific Research (C) No. 25400134. 
assumption on the history-responsive operator $F$ to be globally defined and globally Lipschitz continuous, or, locally Lipschitz continuous on each bounded subset of $\mathfrak{X}$. The assumption was made in previous works in order to apply the wellknown generation theorem of Crandall-Liggett [3] to the operator $\mathfrak{A}$ in $\mathfrak{X}$ defined by $\mathfrak{A} \phi=\phi^{\prime}$ for $\phi \in \mathfrak{X}_{0}$ satisfying $\phi^{\prime} \in \mathfrak{X}, \phi(0) \in D(A)$ and $\phi^{\prime}(0) \in A \phi(0)+F(\phi)$.

In [24, in addition to the dissipativity condition (A5) on $F$ in Section 2, it was assumed instead of the global Lipschitz condition on $F$ that there exists $L_{F}>0$ such that $\|F(\phi)-F(\hat{\phi})\|_{X} \leq L_{F}\|\phi-\hat{\phi}\|_{\mathfrak{X}}$ for $\phi, \hat{\phi} \in \mathfrak{X}_{0}$ with $\phi(0)=\hat{\phi}(0)$. This condition was used to show that the operator $\mathfrak{A}$ satisfies the subtangential condition in the sense of Pierre [19.

The purpose is to prove that the main result in [24] remains true without the above-mentioned assumption. The strategy is that we directly construct a family of approximate solutions and show the convergence of a family of approximate solutions by Kobayashi's argument ([11]), without obtaining a representation theorem for solutions of (FDE) as a quasi-contractive semigroup on $\mathfrak{D}$. In this sense, our approach to the flow invariance problem for (FDE) is completely different from the previous works mentioned above.

In Section 2 we introduce an initial-history space $\mathfrak{X}$ and assumptions on the operators $A$ and $F$ and the set $\mathfrak{D}$ appearing in (FDE), and state the main results concerning the flow invariance problem for (FDE). Moreover, we present a result for the continuous dependence of mild solutions to (FDE) on initial values and a solution to the problem of whether any differentiable mild solution satisfies the equation (FDE). Section 3 contains the proof of Theorem 2.3. Section 4 is devoted to the proof of Theorem 2.6 together with a key inequality concerning the uniqueness of mild solutions of functional differential equations. Here the author would like to express appreciation to the referee for suggesting how best to improve the original manuscript.

\section{MAin RESUlts}

Let $\left(X,\|\cdot\|_{X}\right)$ be a Banach space and let $J$ be an interval of the form $(-\infty, 0]$ or $[-R, 0]$, where $R$ is a positive number. According to the device of Ruess [24], we use the initial-history space $\left(\mathfrak{X},\|\cdot\|_{\mathfrak{X}}\right)$ defined as a Banach space consisting of continuous functions from $J$ into $X$ satisfying the following conditions:

$(\mathfrak{X} 1)$ For any $\phi \in \mathfrak{X},\|\phi(0)\|_{X} \leq\|\phi\|_{\mathfrak{X}}$.

$(\mathfrak{X} 2)$ If $\phi \in \mathfrak{X}$ and $\left\{\phi_{n}\right\}$ is a sequence in $\mathfrak{X}$ such that $\lim _{n \rightarrow \infty}\left\|\phi_{n}-\phi\right\|_{\mathfrak{X}}=0$, then $\lim _{n \rightarrow \infty}\left\|\phi_{n}(\theta)-\phi(\theta)\right\|_{X}=0$ for $\theta \in J$, and

$$
\lim _{n \rightarrow \infty} \int_{\alpha}^{\beta} \phi_{n}(\theta) d \theta=\int_{\alpha}^{\beta} \phi(\theta) d \theta \quad \text { for } \alpha, \beta \in J .
$$

(X3) For any $\lambda>0, x \in X$ and $\phi \in \mathfrak{X}$, the solution $\varphi_{\lambda, x}^{\phi} \in C^{1}(J ; X)$ of the equation $\varphi-\lambda \varphi^{\prime}=\phi$ with $\varphi(0)=x$ is an element of $\mathfrak{X}$ and

$$
\left\|\varphi_{\lambda, x}^{\phi}\right\|_{\mathfrak{X}} \leq \max \left\{\|x\|_{X},\|\phi\|_{\mathfrak{X}}\right\} .
$$

$(\mathfrak{X} 4)$ If $u$ is a continuous function from $J \cup[0, \infty)$ into $X$ and the restriction of $u$ to $J$ is an element of $\mathfrak{X}$, then $u_{t} \in \mathfrak{X}$ for $t \geq 0$, and the mapping $t \rightarrow u_{t}$ is continuous from $[0, \infty)$ into $\mathfrak{X}$.

Remark 2.1. The initial-history space introduced above is different from the original one in that additional conditions were required in [24]. 
Let $D$ be a closed subset of $X$ such that $D(A) \cap D \neq \emptyset$ and let $\mathfrak{X}_{0}=\{\phi \in$ $\mathfrak{X} ; \phi(0) \in \overline{D(A)} \cap D$, where $\overline{D(A)}$ is the closure of $D(A)$ in $X$. We consider the following assumptions on the operators $A$ and $F$ and the set $\mathfrak{D}$ appearing in (FDE):

(A1) There exists $\omega_{A} \geq 0$ such that $A-\omega_{A} I$ is a dissipative operator in $X$, where $I$ is the identity operator on $X$.

(A2) $\mathfrak{D}$ is a closed subset of $\mathfrak{X}_{0}$.

(A3) If $x \in D(A) \cap D, \phi \in \mathfrak{D}$ and $\lambda>0$ is sufficiently small, then $\varphi_{\lambda, x}^{\phi} \in \mathfrak{D}$.

(A4) $F$ is a continuous operator from $\mathfrak{D}$ into $X$.

(A5) There exists $\omega_{F} \geq 0$ such that

$$
[\phi(0)-\hat{\phi}(0), F(\phi)-F(\hat{\phi})]_{+} \leq \omega_{F}\|\phi-\hat{\phi}\|_{\mathfrak{X}}
$$

for $\phi, \hat{\phi} \in \mathfrak{D}$ satisfying that $\|\phi-\hat{\phi}\|_{\mathfrak{X}} \leq\|\phi(0)-\hat{\phi}(0)\|_{X}$, where

$$
[x, \xi]_{+}=\lim _{h \rightarrow 0+}\left(\|x+h \xi\|_{X}-\|x\|_{X}\right) / h \quad \text { for } x, \xi \in X .
$$

Remark 2.2. In the special case where $\mathfrak{D}=\mathfrak{X}_{0}$, condition (A3) is automatically satisfied.

We discuss the existence and uniqueness of mild solutions to (FDE), where by a mild solution to (FDE; $\phi)$ we mean a continuous function $u$ from $J \cup[0, \infty)$ into $X$ such that $u(t)=\phi(t)$ for $t \in J$ and such that for each $\tau>0$, the restriction of $u$ to $[0, \tau]$ is a mild solution to the Cauchy problem

$(\mathrm{CP} ; x, f) \quad u^{\prime}(t) \in A u(t)+f(t) \quad$ for $t \in[0, \tau], \quad$ and $\quad u(0)=x$

with $x=\phi(0)$ and $f(t)=F\left(u_{t}\right)$ for $t \in[0, \tau]$. We refer the reader to [17] for information on mild solutions to (CP).

The main result in this paper is given by

Theorem 2.3. Let $\mathfrak{X}$ be an initial-history space satisfying conditions $(\mathfrak{X} 1)$ through $(\mathfrak{X} 4)$. Assume conditions (A1) through (A5) to be satisfied, and suppose that

$$
\liminf _{h \rightarrow 0+} h^{-1} d(\phi(0)+h F(\phi),(I-h A)(D(A) \cap D))=0 \quad \text { for } \phi \in \mathfrak{D} .
$$

Then, for any $\phi_{0} \in \mathfrak{D}$ the $\left(\mathrm{FDE} ; \phi_{0}\right)$ has a mild solution $u$ such that $u_{t} \in \mathfrak{D}$ for $t \geq 0$. Here and subsequently, the symbol $d(x, C)$ denotes the distance from $x \in X$ to a subset $C$ of $X$.

Remark 2.4. Theorem 2.3 is an improvement of the main theorem in 24. In fact, the set $\overline{D(A) \cap D}$ was taken in the definition of $\mathfrak{X}_{0}$, although the set $\overline{D(A)} \cap D$ is the largest one of initial values. Moreover, the operator $F$ was assumed to satisfy that there exists $L_{F}>0$ such that $\|F(\phi)-F(\hat{\phi})\|_{X} \leq L_{F}\|\phi-\hat{\phi}\|_{\mathfrak{X}}$ for $\phi, \hat{\phi} \in \mathfrak{X}_{0}$ with $\phi(0)=\hat{\phi}(0)$.

In order to discuss the uniqueness of mild solutions, we consider the following additional assumption on the initial-history space $\mathfrak{X}$ :

$(\mathfrak{X} 5)$ For any continuous function $v$ from $J \cup[0, \infty)$ into $X$ such that the restriction of $v$ to $J$ is an element of $\mathfrak{X}$,

$$
\left\|v_{t}\right\|_{\mathfrak{X}} \leq \max \left(\left\|v_{0}\right\|_{\mathfrak{X}}, \sup \left\{\|v(s)\|_{X} ; 0 \leq s \leq t\right\}\right) \quad \text { for } t \geq 0 .
$$

Remark 2.5. Assumption $(\mathfrak{X} 5)$ on the initial-history space $\mathfrak{X}$ is satisfied for the space $\mathfrak{X}=C([-R, 0] ; X)$ with theusual sup-norm and the weighted sup-norm 
space $\mathfrak{X}=\{\phi \in C((-\infty, 0] ; X) ; w \phi \in B U C((-\infty, 0] ; X)\}$ with norm $\|\phi\|_{\mathfrak{X}}=$ $\sup \left\{w(\theta)\|\phi(\theta)\|_{X} ; \theta \in(-\infty, 0]\right\}$, where $w$ is a weight function from $(-\infty, 0]$ into $(0,1]$ satisfying the following conditions:

(w1) $w$ is continuous, nondecreasing and $w(0)=1$,

(w2) $\lim _{s \rightarrow 0-} w(\theta+s) / w(\theta)=1$ uniformly with respect to $\theta \in(-\infty, 0]$.

The weighted sup-norm space mentioned above is found in [24].

We are now in a position to state the uniqueness result of mild solutions to (FDE).

Theorem 2.6. Let $\mathfrak{X}$ be an initial-history space satisfying conditions (X1) through $(\mathfrak{X} 5)$. Assume conditions (A1) through (A5) to be satisfied. Let $\phi, \hat{\phi} \in \mathfrak{X}$, and let $u$ and $\hat{u}$ be mild solutions to (FDE; $\phi)$ and (FDE; $\hat{\phi})$ respectively. Then,

$$
\|u(t)-\hat{u}(t)\|_{X} \leq e^{\omega t}\|\phi-\hat{\phi}\|_{\mathfrak{X}} \quad \text { for } t \geq 0 .
$$

Remark 2.7. It may be proved by the arguments in 24 that Theorem 2.6 is also true if the following assumptions are made instead of conditions (A5) and ( $\mathfrak{X} 5)$ :

(R1) There exist $M_{0} \geq 1$ and a locally bounded function $M_{1}$ from $[0, \infty)$ into itself such that if $v$ is a continuous function from $J \cup[0, \infty)$ into $X$ and if the restriction of $v$ to $J$ is an element of $\mathfrak{X}$, then

$$
\left\|v_{t}\right\|_{\mathfrak{X}} \leq M_{0}\left\|v_{0}\right\|_{\mathfrak{X}}+M_{1}(t) \sup \left\{\|v(s)\|_{X} ; 0 \leq s \leq t\right\} \quad \text { for } t \geq 0 .
$$

(R2) There exists $M_{F}>0$ such that

$$
[\phi(0)-\hat{\phi}(0), F(\phi)-F(\hat{\phi})]_{+} \leq M_{F}\|\phi-\hat{\phi}\|_{\mathfrak{X}} \quad \text { for } \phi, \hat{\phi} \in \mathfrak{D} .
$$

We conclude this section with remarks. (i) An affirmative answer to the question whether any differentiable mild solution satisfies the equation (FDE) may be given as an analogue of the result of Kobayashi [12, Proposition 5.7] on an evolution equation of the form $u^{\prime}(t) \in A u(t)$ which is an improvement of the result of Miyadera [16, Theorem 1]. (ii) It is should be noted that [24, Theorem 4.5] gives conditions under which a mild solution is a classical solution to (FDE).

\section{Proof of Theorem 2.3}

The strategy is to find a solution to (FDE) as the limit function of a family of approximate solutions satisfying

$$
\left(\phi_{k}^{\epsilon}\right)^{\prime}(0) \in A \phi_{k}^{\epsilon}(0)+F\left(\phi_{k}^{\epsilon}\right)+z_{k}^{\epsilon} \quad \text { and } \quad\left\|z_{k}^{\epsilon}\right\|_{X} \leq \epsilon .
$$

We use the subtangential condition (STC) to prove the following lemma which plays a role in constructing such a family of approximate solutions.

Lemma 3.1. For any $\epsilon>0$ and $\phi \in \mathfrak{D}$, there exist $\delta \in(0, \epsilon], \phi_{\delta} \in \mathfrak{D} \cap C^{1}(J ; X)$ and $z_{\delta} \in X$ such that

(i) $\phi_{\delta}(0) \in D(A) \cap D, \phi_{\delta}^{\prime}(0) \in A \phi_{\delta}(0)+F\left(\phi_{\delta}\right)+z_{\delta}$ and $\left\|z_{\delta}\right\|_{X} \leq \epsilon$,

(ii) $\phi_{\delta}^{\prime} \in \mathfrak{X}$ and $\left\|\phi_{\delta}-\delta \phi_{\delta}^{\prime}-\phi\right\|_{\mathfrak{X}} \leq \delta \epsilon$,

(iii) $\left\|\phi_{\delta}-\phi\right\|_{\mathfrak{X}} \leq \epsilon$.

Proof. Let $\epsilon>0$ and $\phi \in \mathfrak{D}$. By condition (STC) there exist a sequence $\left\{h_{n}\right\}$ of positive numbers, a sequence $\left\{x_{n}\right\}$ in $D(A) \cap D$ and a sequence $\left\{\xi_{n}\right\}$ in $X$ such that $\lim _{n \rightarrow \infty} h_{n}=0, \xi_{n} \in A x_{n}$ for $n \geq 1$, and

$$
\lim _{n \rightarrow \infty} h_{n}^{-1}\left\|\phi(0)+h_{n} F(\phi)-\left(x_{n}-h_{n} \xi_{n}\right)\right\|_{X}=0 .
$$


Set $\phi_{n}=\varphi_{h_{n}, x_{n}}^{\phi}$ for $n \geq 1$. By condition (A3) we have $\phi_{n} \in \mathfrak{D} \cap C^{1}(J ; X)$, $\phi_{n}(0)=x_{n} \in D(A) \cap D$ and $\phi_{n}-h_{n} \phi_{n}^{\prime}=\phi$ for sufficiently large $n \geq 1$. Clearly, $\phi_{n}^{\prime}=h_{n}^{-1}\left(\phi_{n}-\phi\right) \in \mathfrak{X}$ for $n \geq 1$. Set $w_{n}=\left(x_{n}-\phi(0)\right) / h_{n}-\xi_{n}-F(\phi)$ for $n \geq 1$. Then we have $\lim _{n \rightarrow \infty}\left\|w_{n}\right\|_{X}=0$ by (3.2). Set $z_{n}=w_{n}+F(\phi)-F\left(\phi_{n}\right)$ for $n \geq 1$. Then we have $\phi_{n}^{\prime}(0)=\left(x_{n}-\phi(0)\right) / h_{n} \in A \phi_{n}(0)+F\left(\phi_{n}\right)+z_{n}$ for $n \geq 1$. As in Step 2.1 of the proof of 24, Theorem 2.1] we find that $\phi_{n} \rightarrow \phi$ in $\mathfrak{X}$ as $n \rightarrow \infty$. The proof is completed since $\lim _{n \rightarrow \infty}\left\|z_{n}\right\|_{X}=0$ by condition (A4).

Remark 3.2. From Lemma 3.1 we observe that the set $\mathfrak{C}_{0}$ of all $\psi \in \mathfrak{D} \cap C^{1}(J ; X)$ such that $\psi(0) \in D(A), \psi^{\prime} \in \mathfrak{X}$ and $\psi^{\prime}(0) \in A \psi(0)+F(\psi)+z$ for some $z \in X$ is dense in $\mathfrak{D}$.

We need to estimate the difference between two approximate solutions satisfying (3.1) in proving the convergence of a family of approximate solutions. The following lemma will be used for that purpose.

Lemma 3.3. Assume that $\phi, \hat{\phi} \in \mathfrak{D} \cap C^{1}(J ; X), \phi^{\prime}, \hat{\phi}^{\prime} \in \mathfrak{X}$ and $z, \hat{z} \in X$ satisfy

$$
\begin{array}{lll}
\phi(0) \in D(A) & \text { and } & \phi^{\prime}(0) \in A \phi(0)+F(\phi)+z, \\
\hat{\phi}(0) \in D(A) & \text { and } & \hat{\phi}^{\prime}(0) \in A \hat{\phi}(0)+F(\hat{\phi})+\hat{z} .
\end{array}
$$

Then,

$$
\begin{aligned}
& \left(\|\phi-\hat{\phi}\|_{\mathfrak{X}}-\left\|\phi-h \phi^{\prime}-\left(\hat{\phi}-h \hat{\phi}^{\prime}\right)\right\|_{\mathfrak{X}}\right) / h \\
& \quad \leq \omega\|\phi-\hat{\phi}\|_{\mathfrak{X}}+\|z-\hat{z}\|_{X} \quad \text { for } h>0, \\
& \left(\|\phi-\hat{\phi}\|_{\mathfrak{X}}-\left\|\left(\phi-\lambda \phi^{\prime}\right)-\hat{\phi}\right\|_{\mathfrak{X}}\right) / \lambda+\left(\|\phi-\hat{\phi}\|_{\mathfrak{X}}-\left\|\phi-\left(\hat{\phi}-\mu \hat{\phi}^{\prime}\right)\right\|_{\mathfrak{X}}\right) / \mu \\
& \quad \leq \omega\|\phi-\hat{\phi}\|_{\mathfrak{X}}+\|z-\hat{z}\|_{X} \quad \text { for } \lambda, \mu>0,
\end{aligned}
$$

where $\omega=\omega_{A}+\omega_{F}$.

Proof. The inequality (3.4) is obtained by setting $h=\lambda \mu /(\lambda+\mu)$ in (3.3). To prove (3.3), let $h>0$, and set $\psi=\phi-h \phi^{\prime}$ and $\hat{\psi}=\hat{\phi}-h \hat{\phi}^{\prime}$. Since $\psi-\hat{\psi}=$ $(\phi-\hat{\phi})-h(\phi-\hat{\phi})^{\prime} \in \mathfrak{X}$, we have $\|\phi-\hat{\phi}\|_{\mathfrak{X}} \leq \max \left\{\|\phi(0)-\hat{\phi}(0)\|_{X},\|\psi-\hat{\psi}\|_{\mathfrak{X}}\right\}$ by condition ( $\mathfrak{X} 3)$. The inequality (3.3) is clearly true if $\|\phi(0)-\hat{\phi}(0)\|_{X} \leq\|\psi-\hat{\psi}\|_{\mathfrak{X}}$. Now, we consider the case where $\|\phi(0)-\hat{\phi}(0)\|_{X} \geq\|\psi-\hat{\psi}\|_{\mathfrak{X}}$. In this case, we note that $\|\phi-\hat{\phi}\|_{\mathfrak{X}}=\|\phi(0)-\hat{\phi}(0)\|_{X}$ by condition $(\mathfrak{X} 1)$. By condition (A5) we have $[\phi(0)-\hat{\phi}(0), F(\phi)-F(\hat{\phi})]_{+} \leq \omega_{F}\|\phi-\hat{\phi}\|_{\mathfrak{X}}$. By condition (A1) we have $\left[\phi(0)-\hat{\phi}(0), \phi^{\prime}(0)-F(\phi)-z-(\hat{\phi}(0)-F(\hat{\phi})-\hat{z})\right]_{-} \leq \omega_{A}\|\phi(0)-\hat{\phi}(0)\|_{X}$, where $[x, \xi]_{-}=\lim _{h \rightarrow 0+}\left(\|x\|_{X}-\|x-h \xi\|_{X}\right) / h$ for $x, \xi \in X$. Since $\phi^{\prime}(0)=(\phi(0)-\psi(0)) / h$ and $\hat{\phi}^{\prime}(0)=(\hat{\phi}(0)-\hat{\psi}(0)) / h$, we find that

$$
\begin{aligned}
& \left(\|\phi(0)-\hat{\phi}(0)\|_{X}-\|\psi(0)-\hat{\psi}(0)\|_{X}\right) / h \\
& \quad \leq \omega_{A}\|\phi(0)-\hat{\phi}(0)\|_{X}+[\phi(0)-\hat{\phi}(0), F(\phi)-F(\hat{\phi})]_{+}+\|z-\hat{z}\|_{X} .
\end{aligned}
$$

The desired inequality (3.3) is obtained by combining the above inequalities and the inequality that $\|\psi(0)-\hat{\psi}(0)\|_{X} \leq\|\psi-\hat{\psi}\|_{\mathfrak{X}}$ by condition $(\mathfrak{X} 1)$. 
The construction of a family of approximate solutions is provided by

Lemma 3.4. Let $\epsilon>0$ and $\phi_{0} \in \mathfrak{D}$. Then there exist a sequence $\left\{t_{k}\right\}_{k=0}^{\infty}$ of nonnegative numbers, a sequence $\left\{\phi_{k}\right\}_{k=1}^{\infty}$ in $\mathfrak{D} \cap C^{1}(J ; X)$ and a sequence $\left\{z_{k}\right\}_{k=1}^{\infty}$ in $X$ such that

(i) $0=t_{0}<t_{1}<\cdots<t_{k}<\cdots$,

(ii) $t_{k}-t_{k-1} \leq \epsilon$ for $k=1,2,3, \ldots$,

(iii) $\phi_{k}(0) \in D(A), \phi_{k}^{\prime}(0) \in A \phi_{k}(0)+F\left(\phi_{k}\right)+z_{k}$ and $\left\|z_{k}\right\|_{X} \leq \epsilon$ for $k=$ $1,2,3, \ldots$,

(iv) $\phi_{k}^{\prime} \in \mathfrak{X}$ and $\left\|\phi_{k}-\left(t_{k}-t_{k-1}\right) \phi_{k}^{\prime}-\phi_{k-1}\right\|_{\mathfrak{X}} \leq\left(t_{k}-t_{k-1}\right) \epsilon$ for $k=1,2,3, \ldots$,

(v) $\lim _{k \rightarrow \infty} t_{k}=\infty$.

Proof. Let $\epsilon>0$. For $\phi \in \mathfrak{D}$ we define $\delta(\phi)$ by the supremum of the set $S(\phi)$ consisting of all $\delta \in(0, \epsilon]$ such that there exist $\phi_{\delta} \in \mathfrak{D} \cap C^{1}(J ; X)$ and $z_{\delta} \in X$ satisfying conditions (i) and (ii) in Lemma 3.1. By Lemma 3.1 we have $\delta(\phi)>0$ for any $\phi \in \mathfrak{D}$. Now, let $\phi_{0} \in \mathfrak{D}$ and $t_{0}=0$. Let $l \geq 1$ and assume that $\left\{t_{k}\right\}_{k=0}^{l-1}$, $\left\{\phi_{k}\right\}_{k=0}^{l-1}$ and $\left\{z_{k}\right\}_{k=1}^{l-1}$ are already chosen as required. Since $\phi_{l-1} \in \mathfrak{D}$, we have $\delta\left(\phi_{l-1}\right)>0$, and so there exists $h_{l} \in S\left(\phi_{l-1}\right)$ such that $\delta\left(\phi_{l-1}\right) / 2<h_{l}$. Since $h_{l} \in S\left(\phi_{l-1}\right)$, we observe that $h_{l} \in(0, \epsilon]$ and there exist $\phi_{l} \in \mathfrak{D} \cap C^{1}(J ; X)$ and $z_{l} \in X$ such that $\phi_{l}(0) \in D(A), \phi_{l}^{\prime}(0) \in A \phi_{l}(0)+F\left(\phi_{l}\right)+z_{l},\left\|z_{l}\right\|_{X} \leq \epsilon, \phi_{l}^{\prime} \in \mathfrak{X}$ and $\left\|\phi_{l}-h_{l} \phi_{l}^{\prime}-\phi_{l-1}\right\|_{\mathfrak{X}} \leq h_{l} \epsilon$. Set $t_{l}=t_{l-1}+h_{l}$. Then, conditions (ii) through (iv) are clearly satisfied for $k=l$. To verify condition (v), assume to the contrary that $\lim _{k \rightarrow \infty} t_{k}=: \bar{t}<\infty$. Then, there exists $\bar{\phi} \in \mathfrak{X}$ such that $\phi_{k} \rightarrow \bar{\phi}$ in $\mathfrak{X}$ as $k \rightarrow \infty$. This fact will be shown in the last part. Since $\mathfrak{D}$ is closed in $\mathfrak{X}$, we have $\bar{\phi} \in \mathfrak{D}$. By Lemma 3.1 there exist $\delta \in(0, \epsilon / 2], \phi_{\delta} \in \mathfrak{D} \cap C^{1}(J ; X)$ and $z_{\delta} \in X$ such that $\phi_{\delta}(0) \in D(A) \cap D, \phi_{\delta}^{\prime}(0) \in A \phi_{\delta}(0)+F\left(\phi_{\delta}\right)+z_{\delta},\left\|z_{\delta}\right\|_{X} \leq \epsilon / 2, \phi_{\delta}^{\prime} \in \mathfrak{X}$ and $\left\|\phi_{\delta}-\delta \phi_{\delta}^{\prime}-\bar{\phi}\right\|_{\mathfrak{X}} \leq \delta \epsilon / 2$. Since $\phi_{l} \rightarrow \bar{\phi}$ in $\mathfrak{X}$ as $l \rightarrow \infty$, we have $\left\|\phi_{\delta}-\delta \phi_{\delta}^{\prime}-\phi_{l-1}\right\|_{\mathfrak{X}} \leq \delta \epsilon$ for sufficiently large $l \geq 1$; hence $\delta \leq \delta\left(\phi_{l-1}\right)$ for sufficiently large $l \geq 1$. This a contradiction to the fact that $\delta\left(\phi_{l-1}\right)<2 h_{l} \rightarrow 0$ as $l \rightarrow \infty$.

Finally, we prove that if $t_{k} \rightarrow \bar{t}<\infty$ as $k \rightarrow \infty$, then the sequence $\left\{\phi_{k}\right\}$ converges in $\mathfrak{X}$ as $k \rightarrow \infty$. For this purpose, let $m \geq 1$ be an integer such that $\omega h_{p}<1$ for $p \geq m$, and it suffices to show that

$$
\alpha_{k, l}\left\|\phi_{k}-\phi_{l}\right\|_{\mathfrak{X}} \leq\left(t_{k}-t_{l}\right)\left(\left\|\phi_{m}^{\prime}\right\|_{\mathfrak{X}}+\epsilon\right)+2\left(t_{k}-t_{m}\right) \epsilon+2\left(t_{l}-t_{m}\right) \epsilon
$$

for $k \geq l \geq m$. Here $\alpha_{k, l}=\prod_{p=m+1}^{k}\left(1-\omega h_{p}\right) \prod_{p=m+1}^{l}\left(1-\omega h_{p}\right)$ for $k \geq l \geq$ $m$, where $\prod_{p=m+1}^{m}\left(1-\omega h_{p}\right)=1$. By (3.3) we have $\left(1-h_{k} \omega\right)\left\|\phi_{k}-\phi_{m}\right\|_{\mathfrak{X}} \leq$ $\left\|\phi_{k-1}-\phi_{m}\right\|_{\mathfrak{X}}+h_{k}\left(\left\|\phi_{m}^{\prime}\right\|_{\mathfrak{X}}+\epsilon\right)+2 h_{k} \epsilon$ for $k \geq m+1$, where we have used that $\left\|\phi_{k}-h_{k} \phi_{k}^{\prime}-\phi_{k-1}\right\|_{\mathfrak{X}} \leq h_{k} \epsilon$ for $k \geq m+1$. Thus, we can inductively verify (3.5) for $l=m$. Clearly, the inequality (3.5) is true for $k=l$. By (3.4) we find that

$$
\begin{aligned}
& \left(\left\|\phi_{k}-\phi_{l}\right\|_{\mathfrak{X}}-\left\|\phi_{k-1}-\phi_{l}\right\|_{\mathfrak{X}}\right) / h_{k}+\left(\left\|\phi_{k}-\phi_{l}\right\|_{\mathfrak{X}}-\left\|\phi_{k}-\phi_{l-1}\right\|_{\mathfrak{X}}\right) / h_{l} \\
& \quad \leq \omega\left\|\phi_{k}-\phi_{l}\right\|_{\mathfrak{X}}+4 \epsilon
\end{aligned}
$$

for $k>l>m$. This inequality is an analogue of the inequality in the first line but six from the bottom of page 660 in [11. The inequality (3.5) is demonstrated by Kobayashi's argument [11, Lemma 5.1] (see also the proof of [17, Lemma 5.11]).

The following lemma gives a key estimate on the difference between two approximate solutions. 
Lemma 3.5. Let $\epsilon>0$ and $\phi_{0} \in \mathfrak{D}$. Let $\left\{t_{k}\right\}_{k=0}^{N},\left\{\phi_{k}\right\}_{k=1}^{N}$ and $\left\{z_{k}\right\}_{k=1}^{N}$ be sequences in $[0, \infty), \mathfrak{D} \cap C^{1}(J ; X)$ and $X$ respectively such that

(i) $0=t_{0}<t_{1}<\cdots<t_{N}$,

(ii) $t_{k}-t_{k-1} \leq \epsilon$ for $1 \leq k \leq N$,

(iii) $\phi_{k}(0) \in D(A), \phi_{k}^{\prime}(0) \in \bar{A} \phi_{k}(0)+F\left(\phi_{k}\right)+z_{k}$ and $\left\|z_{k}\right\|_{X} \leq \epsilon$ for $1 \leq k \leq N$,

(iv) $\phi_{k}^{\prime} \in \mathfrak{X}$ and $\left\|\phi_{k}-\left(t_{k}-t_{k-1}\right) \phi_{k}^{\prime}-\phi_{k-1}\right\|_{\mathfrak{X}} \leq\left(t_{k}-t_{k-1}\right) \epsilon$ for $1 \leq k \leq N$.

Let $\hat{\epsilon}>0$ and $\hat{\phi}_{0} \in \mathfrak{D}$, and let $\left\{\hat{t}_{k}\right\}_{k=0}^{\hat{N}},\left\{\hat{\phi}_{k}\right\}_{k=1}^{\hat{N}}$ and $\left\{\hat{z}_{k}\right\}_{k=1}^{\hat{N}}$ be the corresponding ones. Assume that $\omega \epsilon<1$ and $\omega \hat{\epsilon}<1$. Then,

$$
\begin{aligned}
\beta_{k, l}\left\|\phi_{k}-\hat{\phi}_{l}\right\|_{\mathfrak{X}} \leq & \left\|\phi_{0}-\phi\right\|_{\mathfrak{X}}+\left\|\hat{\phi}_{0}-\phi\right\|_{\mathfrak{X}} \\
& +\left\{\left(t_{k}-\hat{t}_{l}\right)^{2}+\epsilon t_{k}+\hat{\epsilon} \hat{t}_{l}\right\}^{1 / 2}\left(\left\|\phi^{\prime}\right\|_{\mathfrak{X}}+\|z\|_{X}\right)+2\left(t_{k} \epsilon+\hat{t}_{l} \hat{\epsilon}\right)
\end{aligned}
$$

for $0 \leq k \leq N, 0 \leq l \leq \hat{N}$, and $\phi \in \mathfrak{C}_{0}$ and $z \in X$ satisfying that $\phi^{\prime}(0) \in A \phi(0)+$ $F(\phi)+z$. Here $\beta_{k, l}=\prod_{p=1}^{k}\left(1-\omega\left(t_{p}-t_{p-1}\right)\right) \prod_{p=1}^{l}\left(1-\omega\left(\hat{t}_{p}-\hat{t}_{p-1}\right)\right)$ for $0 \leq k \leq N$ and $0 \leq l \leq \hat{N}$, where $\prod_{p=1}^{0}\left(1-\omega\left(t_{p}-t_{p-1}\right)\right)=\prod_{p=1}^{0}\left(1-\omega\left(\hat{t}_{p}-\hat{t}_{p-1}\right)\right)=1$, and $\mathfrak{C}_{0}$ is the set defined in Remark 3.2 .

Proof. Let $\phi \in \mathfrak{C}_{0}$ and $z \in X$ satisfy that $\phi^{\prime}(0) \in A \phi(0)+F(\phi)+z$. For simplicity, let $h_{k}=t_{k}-t_{k-1}$ for $1 \leq k \leq N$ and $\hat{h}_{k}=\hat{t}_{k}-\hat{t}_{k-1}$ for $1 \leq k \leq \hat{N}$. Since $\phi_{k}^{\prime}(0) \in$ $A \phi_{k}(0)+F\left(\phi_{k}\right)+z_{k},\left\|z_{k}\right\|_{X} \leq \epsilon$ and $\left\|\phi_{k}-h_{k} \phi_{k}^{\prime}-\phi_{k-1}\right\|_{\mathfrak{X}} \leq h_{k} \epsilon$ for $1 \leq k \leq N$, we find by (3.3) that $\left(1-h_{k} \omega\right)\left\|\phi_{k}-\phi\right\|_{\mathfrak{X}} \leq\left\|\phi_{k-1}-\phi\right\|_{\mathfrak{X}}+h_{k}\left\|\phi^{\prime}\right\|_{\mathfrak{X}}+h_{k}\left(2 \epsilon+\|z\|_{X}\right)$ for $1 \leq k \leq N$. Thus, we inductively prove that $\beta_{k, 0}\left\|\phi_{k}-\phi\right\|_{\mathfrak{X}} \leq\left\|\phi_{0}-\phi\right\|_{\mathfrak{X}}+$ $t_{k}\left(\left\|\phi^{\prime}\right\|_{\mathfrak{X}}+\|z\|_{X}\right)+2 t_{k} \epsilon$ for $0 \leq k \leq N$. This implies that (3.6) holds for $l=0$. Similarly, the inequality (3.6) is shown to be true for $k=0$. By (3.4) we find that

$$
\begin{aligned}
& \left(\left\|\phi_{k}-\hat{\phi}_{l}\right\|_{\mathfrak{X}}-\left\|\phi_{k-1}-\hat{\phi}_{l}\right\|_{\mathfrak{X}}\right) / h_{k}+\left(\left\|\phi_{k}-\hat{\phi}_{l}\right\|_{\mathfrak{X}}-\left\|\phi_{k}-\hat{\phi}_{l-1}\right\|_{\mathfrak{X}}\right) / \hat{h}_{l} \\
& \quad \leq \omega\left\|\phi_{k}-\hat{\phi}_{l}\right\|_{\mathfrak{X}}+2(\epsilon+\hat{\epsilon})
\end{aligned}
$$

for $1 \leq k \leq N$ and $1 \leq l \leq \hat{N}$. This inequality is an analogue of the inequality in line 12 of page 648 in [11. The inequality (3.6) is verified as in the proof of [11. Lemma 2.1] (see also the proof of the inequality (5.15) in [17]).

Proof of Theorem 2.3. Let $\phi_{0} \in \mathfrak{D}$. By Lemma 3.4 we observe that for each $\epsilon>0$ there exist a sequence $\left\{t_{k}^{\epsilon}\right\}_{k=0}^{\infty}$ of nonnegative numbers, a sequence $\left\{\phi_{k}^{\epsilon}\right\}_{k=1}^{\infty}$ in $\mathfrak{D} \cap C^{1}(J ; X)$ and a sequence $\left\{z_{k}^{\epsilon}\right\}_{k=1}^{\infty}$ in $X$ such that

(i) $0=t_{0}^{\epsilon}<t_{1}^{\epsilon}<\cdots<t_{k}^{\epsilon}<\cdots$,

(ii) $t_{k}^{\epsilon}-t_{k-1}^{\epsilon} \leq \epsilon$ for $k=1,2,3, \ldots$,

(iii) $\phi_{k}^{\epsilon}(0) \in D(A),\left(\phi_{k}^{\epsilon}\right)^{\prime}(0) \in A \phi_{k}^{\epsilon}(0)+F\left(\phi_{k}^{\epsilon}\right)+z_{k}^{\epsilon}$ and $\left\|z_{k}^{\epsilon}\right\|_{X} \leq \epsilon$ for $k=$ $1,2,3, \ldots$

(iv) $\left(\phi_{k}^{\epsilon}\right)^{\prime} \in \mathfrak{X}$ and $\left\|\phi_{k}^{\epsilon}-\left(t_{k}^{\epsilon}-t_{k-1}^{\epsilon}\right)\left(\phi_{k}^{\epsilon}\right)^{\prime}-\phi_{k-1}^{\epsilon}\right\|_{\mathfrak{X}} \leq\left(t_{k}^{\epsilon}-t_{k-1}^{\epsilon}\right) \epsilon$ for $k=$ $1,2,3, \ldots$, where $\phi_{0}^{\epsilon}=\phi_{0}$,

(v) $\lim _{k \rightarrow \infty} t_{k}^{\epsilon}=\infty$.

For each $\epsilon>0$, we define a function $\phi^{\epsilon}:[0, \infty) \rightarrow \mathfrak{X}$ by

$$
\phi^{\epsilon}(t)= \begin{cases}\phi_{0}^{\epsilon} & \text { for } t=0, \\ \phi_{k}^{\epsilon} & \text { for } t \in\left(t_{k-1}^{\epsilon}, t_{k}^{\epsilon}\right] \text { and } k=1,2,3, \ldots\end{cases}
$$

Since the set $\mathfrak{C}_{0}$ is dense in $\mathfrak{D}$ (see Remark 3.2 ), we deduce from Lemma 3.5 that there exists $\phi \in C([0, \infty) ; \mathfrak{X})$ such that

$$
\lim _{\epsilon \rightarrow 0+} \phi^{\epsilon}(t)=\phi(t) \quad \text { for } t \geq 0,
$$


where the limit is uniform on any compact subinterval of $[0, \infty)$. By $\phi(t, \theta)$ we denote the value of $\phi(t) \in \mathfrak{X}$ at $\theta \in J$. By condition $(\mathfrak{X} 2)$ we observe that the function $u$ from $J \cup[0, \infty)$ into $X$, defined by $u(t)=\phi_{0}(t)$ for $t \in J$, and $u(t)=$ $\phi(t, 0)$ for $t \geq 0$, is continuous in $X$.

Let $\tau>0$ and we prove that the restriction of $u$ to $[0, \tau]$ is a mild solution to $\left(\mathrm{CP} ; \phi_{0}(0), f\right)$ on $[0, \tau]$, where $f(t)=F(\phi(t))$ for $t \in[0, \tau]$. For each $\epsilon>0$, set $N^{\epsilon}=\max \left\{k ; t_{k}^{\epsilon} \leq \tau\right\}$, and let $u_{k}^{\epsilon}=\phi_{k}^{\epsilon}(0)$ for $0 \leq k \leq N^{\epsilon}$ and $f_{k}^{\epsilon}=F\left(\phi_{k}^{\epsilon}\right)+z_{k}^{\epsilon}+$ $\left(\phi_{k}^{\epsilon}(0)-\phi_{k-1}^{\epsilon}(0)\right) /\left(t_{k}^{\epsilon}-t_{k-1}^{\epsilon}\right)-\left(\phi_{k}^{\epsilon}\right)^{\prime}(0)$ for $1 \leq k \leq N^{\epsilon}$. Let $\epsilon>0$. Then we find that $\left(u_{k}^{\epsilon}-u_{k-1}^{\epsilon}\right) /\left(t_{k}^{\epsilon}-t_{k-1}^{\epsilon}\right) \in A u_{k}^{\epsilon}+f_{k}^{\epsilon}$ for $1 \leq k \leq N^{\epsilon}$ and

$$
\sum_{k=1}^{N^{\epsilon}} \int_{t_{k-1}^{\epsilon}}^{t_{k}^{\epsilon}}\left\|f_{k}^{\epsilon}-F(\phi(t))\right\|_{X} d t \leq \int_{0}^{t_{N^{\epsilon}}^{\epsilon}}\left\|F\left(\phi^{\epsilon}(t)\right)-F(\phi(t))\right\|_{X} d t+2 \epsilon t_{N^{\epsilon}}^{\epsilon},
$$

where we have used conditions (iii) and (iv) combined with condition ( $\mathfrak{X} 1$ ). As in the argument at the bottom of page 4381 in 24, the first term on the right-hand side tends to zero as $\epsilon \rightarrow 0+$. Consider the function $u^{\epsilon}:\left[0, t_{N^{\epsilon}}^{\epsilon}\right] \rightarrow X$ defined by

$$
u^{\epsilon}(t)= \begin{cases}u_{0}^{\epsilon} & \text { for } t=0, \\ u_{k}^{\epsilon} & \text { for } t \in\left(t_{k-1}^{\epsilon}, t_{k}^{\epsilon}\right] \text { and } 1 \leq k \leq N^{\epsilon} .\end{cases}
$$

By $\phi^{\epsilon}(t, \theta)$ we denote the value of $\phi^{\epsilon}(t) \in \mathfrak{X}$ at $\theta \in J$. Since $\left\|u^{\epsilon}(t)-u(t)\right\|_{X}=$ $\left\|\phi^{\epsilon}(t, 0)-\phi(t, 0)\right\|_{\mathfrak{X}}$ for $t \in\left[0, t_{N^{\epsilon}}^{\epsilon}\right]$, it follows from (3.8) and condition (X1) that $\lim _{\epsilon \rightarrow 0+}\left(\sup \left\{\left\|u^{\epsilon}(t)-u(t)\right\|_{X} ; t \in\left[0, t_{N^{\epsilon}}^{\epsilon}\right]\right\}\right)=0$. Therefore, we see that the restriction of $u$ to $[0, \tau]$ is a mild solution to $\left(\mathrm{CP} ; \phi_{0}(0), f\right)$ on $[0, \tau]$, where $f(t)=F(\phi(t))$ for $t \in[0, \tau]$. The fact that $\phi(t)=u_{t}$ for $t \geq 0$ is proved by Plant's argument ([20, Theorem 3.1]). Therefore, the proof is completed.

\section{Proof of Theorem 2.6}

To prove the uniqueness of mild solutions to (FDE), we need the following inequality concerning functional differential equations.

Lemma 4.1. Let $m \in C(J \cup[0, \infty) ; X)$ and $\beta \geq 0$. Assume that the restriction of $m$ to $J$ is an element of $\mathfrak{X}$ and that

$$
D^{+}\|m(t)\|_{X} \leq \beta\|m(t)\|_{X}
$$

for all $t \geq 0$ satisfying that $\left\|m_{t}\right\|_{\mathfrak{X}} \leq\|m(t)\|_{X}$, where

$$
D^{+}\|m(t)\|_{X}=\limsup _{h \rightarrow 0+}\left(\|m(t+h)\|_{X}-\|m(t)\|_{X}\right) / h
$$

for $t \geq 0$. Then,

$$
\|m(t)\|_{X} \leq e^{\beta t}\left\|m_{0}\right\|_{\mathfrak{X}} \quad \text { for } t \geq 0 .
$$

Proof. For any $\epsilon>0$, it suffices to prove that

$$
\|m(t)\|_{X} \leq e^{(\beta+\epsilon) t}\left(\left\|m_{0}\right\|_{\mathfrak{X}}+\epsilon\right) \quad \text { for } t \geq 0 .
$$

Let $\epsilon>0$ and assume that (4.2) is not true. Then, the set $Z$ defined by

$$
Z=\left\{t \geq 0 ;\|m(t)\|_{X}>e^{(\beta+\epsilon) t}\left(\left\|m_{0}\right\|_{\mathfrak{X}}+\epsilon\right)\right\}
$$

is nonempty, and set $t_{0}=\inf Z$. Since $\|m(0)\|_{X} \leq\left\|m_{0}\right\|_{\mathfrak{X}}$ by condition $(\mathfrak{X} 1)$, we find that $t_{0}>0$ and

$$
\begin{aligned}
& \left\|m\left(t_{0}\right)\right\|_{X}=e^{(\beta+\epsilon) t_{0}}\left(\left\|m_{0}\right\|_{\mathfrak{X}}+\epsilon\right), \\
& \|m(t)\|_{X} \leq e^{(\beta+\epsilon) t}\left(\left\|m_{0}\right\|_{\mathfrak{X}}+\epsilon\right) \text { for } 0 \leq t<t_{0} .
\end{aligned}
$$


It follows from condition $(\mathfrak{X} 5)$ that $\left\|m_{t_{0}}\right\|_{\mathfrak{X}} \leq e^{(\beta+\epsilon) t_{0}}\left(\left\|m_{0}\right\|_{\mathfrak{X}}+\epsilon\right)=\left\|m\left(t_{0}\right)\right\|_{X}$. Therefore, we use the assumption (4.1) to obtain

$$
D^{+}\left\|m\left(t_{0}\right)\right\|_{X} \leq \beta\left\|m\left(t_{0}\right)\right\|_{X} .
$$

By the definition of $t_{0}$, there exists a sequence $\left\{t_{n}\right\}$ in $Z$ such that $t_{n} \rightarrow t_{0}$ as $n \rightarrow \infty$ and such that

$$
\left\|m\left(t_{n}\right)\right\|_{X}>e^{(\beta+\epsilon) t_{n}}\left(\left\|m_{0}\right\|_{\mathfrak{X}}+\epsilon\right)
$$

for $n \geq 1$. By (4.3) and (4.4) we note that $t_{n}>t_{0}$ for $n \geq 1$. Subtracting (4.3) from (4.6), and letting $n \rightarrow \infty$ in the inequality divided by $t_{n}-t_{0}$, we find that $D^{+}\left\|m\left(t_{0}\right)\right\|_{X} \geq(\beta+\epsilon) e^{(\beta+\epsilon) t_{0}}\left(\left\|m_{0}\right\|_{\mathfrak{X}}+\epsilon\right)=(\beta+\epsilon)\left\|m\left(t_{0}\right)\right\|_{X}$ by (4.3). This is a contradiction to (4.5).

Proof of Theorem 2.6. Let $\phi, \hat{\phi} \in \mathfrak{X}$, and let $u$ and $\hat{u}$ be mild solutions to (FDE; $\phi$ ) and $(\mathrm{FDE} ; \hat{\phi})$ respectively. Note that for $\tau>0$ the restriction of $u$ to $[0, \tau]$ is a mild solution on $[0, \tau]$ to $(\mathrm{CP} ; \phi(0), f)$, where $f(t)=F\left(u_{t}\right)$ for $t \in[0, \tau]$, and so it is also an integral solution on $[0, \tau]$ to $(\mathrm{CP} ; \phi(0), f)$. By [1,17] we have

$$
\begin{aligned}
& e^{-\omega_{A} t}\|u(t)-\hat{u}(t)\|_{X}-e^{-\omega_{A} s}\|u(s)-\hat{u}(s)\|_{X} \\
& \quad \leq \int_{s}^{t} e^{-\omega_{A} \sigma}\left[u(\sigma)-\hat{u}(\sigma), F\left(u_{\sigma}\right)-F\left(\hat{u}_{\sigma}\right)\right]_{+} d \sigma
\end{aligned}
$$

for $t \geq s \geq 0$. Consider the continuous function $m$ from $J \cup[0, \infty)$ into $X$ defined by $m(t)=u(t)-\hat{u}(t)$ for $t \in J \cup[0, \infty)$. Then, we find by (4.7) that

$$
D^{+}\|m(t)\|_{X} \leq \omega_{A}\|m(t)\|_{X}+\left[u(t)-\hat{u}(t), F\left(u_{t}\right)-F\left(\hat{u}_{t}\right)\right]_{+}
$$

for $t \geq 0$. By condition (A5) we have $D^{+}\|m(t)\|_{X} \leq \omega\|m(t)\|_{X}$ for all $t$ satisfying that $\left\|m_{t}\right\|_{\mathfrak{X}} \leq\|m(t)\|_{X}$. Therefore, we obtain (2.1) by Lemma 4.1.

\section{REFERENCES}

[1] Ph. Bénilan, Equations d'évolution dans un espace de Banach quelconque et applications, Thèsis, Orsay, 1972.

[2] Dennis W. Brewer, A nonlinear semigroup for a functional differential equation, Trans. Amer. Math. Soc. 236 (1978), 173-191. MR0466838 (57 \#6712)

[3] M. G. Crandall and T. M. Liggett, Generation of semi-groups of nonlinear transformations on general Banach spaces, Amer. J. Math. 93 (1971), 265-298. MR0287357 (44 \#4563)

[4] Janet Dyson and Rosanna Villella Bressan, Functional differential equations and non-linear evolution operators, Proc. Roy. Soc. Edinburgh Sect. A 75 (1975/76), no. 3, 223-234. MR.0442402(56 \#784)

[5] Janet Dyson and Rosanna Villella Bressan, Semigroups of translations associated with functional and functional-differential equations, Proc. Roy. Soc. Edinburgh Sect. A $82(1978 / 79)$, no. 3-4, 171-188, DOI 10.1017/S030821050001115X. MR532900 (80i:34113)

[6] H. Flaschka and M. J. Leitman, On semigroups of nonlinear operators and the solution of the functional differential equation $\dot{x}(t)=F\left(x_{t}\right)$, J. Math. Anal. Appl. 49 (1975), 649-658. MR0361959 (50 \#14401)

[7] Jack K. Hale, Functional differential equations with infinite delays, J. Math. Anal. Appl. 48 (1974), 276-283. MR0364813 (51 \#1067)

[8] F. Kappel and W. Schappacher, Some considerations to the fundamental theory of infinite delay equations, J. Differential Equations 37 (1980), no. 2, 141-183, DOI 10.1016/00220396(80)90093-5. MR587220 (81j:34117)

[9] A. G. Kartsatos and M. E. Parrott, Global solutions of functional evolution equations involving locally defined Lipschitzian perturbations, J. London Math. Soc. (2) 27 (1983), no. 2, 306-316, DOI 10.1112/jlms/s2-27.2.306. MR692536 (85g:34066) 
[10] Athanassios G. Kartsatos and Mary E. Parrott, The weak solution of a functional-differential equation in a general Banach space, J. Differential Equations 75 (1988), no. 2, 290-302, DOI 10.1016/0022-0396(88)90140-4. MR961157 (89k:34134)

[11] Yoshikazu Kobayashi, Difference approximation of Cauchy problems for quasi-dissipative operators and generation of nonlinear semigroups, J. Math. Soc. Japan 27 (1975), no. 4, 640-665. MR0399974 (53 \#3812)

[12] Y. Kobayashi, Lectures on Nonlinear Semigroups (in Japanese).

[13] V. Lakshmikantham, S. Leela, and V. Moauro, Existence and uniqueness of solutions of delay differential equations on a closed subset of a Banach space, Nonlinear Anal. 2 (1978), no. 3, 311-327, DOI 10.1016/0362-546X(78)90020-2. MR512662 (80c:34069)

[14] S. Leela and Vinicio Moauro, Existence of solutions in a closed set for delay differential equations in Banach spaces, Nonlinear Anal. 2 (1978), no. 1, 47-58, DOI 10.1016/0362546X(78)90040-8. MR512653 (80c:34070)

[15] James H. Lightbourne III, Function space flow invariance for functional-differential equations of retarded type, Proc. Amer. Math. Soc. 77 (1979), no. 1, 91-98, DOI 10.2307/2042722. MR.539637 (80j:34089)

[16] Isao Miyadera, Some remarks on semi-groups of nonlinear operators, Tôhoku Math. J. (2) 23 (1971), 245-258. MR0296746 (45 \#5805)

[17] Isao Miyadera, Nonlinear semigroups, Translations of Mathematical Monographs, vol. 109, American Mathematical Society, Providence, RI, 1992. Translated from the 1977 Japanese original by Choong Yun Cho. MR.1192132 (93j:47093)

[18] N. Pavel and F. Iacob, Invariant sets for a class of perturbed differential equations of retarded type, Israel J. Math. 28 (1977), no. 3, 254-264. MR0466829 (57 \#6703)

[19] Michel Pierre, Invariant closed subsets for nonlinear semigroups, Nonlinear Anal. 2 (1978), no. 1, 107-117, DOI 10.1016/0362-546X(78)90046-9. MR512659 (80b:47093)

[20] Andrew T. Plant, Nonlinear semigroups of translations in Banach space generated by functional differential equations, J. Math. Anal. Appl. 60 (1977), no. 1, 67-74. MR0447745 (56 \#6055)

[21] Wolfgang M. Ruess, The evolution operator approach to functional-differential equations with delay, Proc. Amer. Math. Soc. 119 (1993), no. 3, 783-791, DOI 10.2307/2160513. MR.1154248 (93m:34132)

[22] W. M. Ruess, Existence of solutions to partial functional-differential equations with delay, Theory and applications of nonlinear operators of accretive and monotone type, Lecture Notes in Pure and Appl. Math., vol. 178, Dekker, New York, 1996, pp. 259-288. MR 1386683 (97b:34085)

[23] Wolfgang M. Ruess, Existence and stability of solutions to partial functional-differential equations with delay, Adv. Differential Equations 4 (1999), no. 6, 843-876. MR1729393 (2000m:34184)

[24] Wolfgang M. Ruess, Flow invariance for nonlinear partial differential delay equations, Trans. Amer. Math. Soc. 361 (2009), no. 8, 4367-4403, DOI 10.1090/S0002-9947-09-04833-8. MR2500891 (2010b:35475)

[25] W. M. Ruess and W. H. Summers, Operator semigroups for functional-differential equations with delay, Trans. Amer. Math. Soc. 341 (1994), no. 2, 695-719, DOI 10.2307/2154579. MR 1214785 (94g:34135)

[26] George Seifert, Positively invariant closed sets for systems of delay differential equations, J. Differential Equations 22 (1976), no. 2, 292-304. MR0427781 (55 \#811)

[27] C. C. Travis and G. F. Webb, Existence and stability for partial functional differential equations, Trans. Amer. Math. Soc. 200 (1974), 395-418. MR.0382808 (52 \#3690)

[28] G. F. Webb, Autonomous nonlinear functional differential equations and nonlinear semigroups, J. Math. Anal. Appl. 46 (1974), 1-12. MR0348224(50 \#722)

Department of Mathematics, Faculty of Science, Shizuoka University, Shizuoka 422 8529, JAPAN

E-mail address: tanaka.naoki@shizuoka.ac.jp 